\title{
Special Feature on Regional Sustainability: analysis in a spatial and regional context with broad perspectives on the risk of global warming, natural disasters, and emerging issues due to the globalized economy
}

\author{
Yoshiro Higano $^{1}$ - Akihiro Otsuka ${ }^{2}$
}

Published online: 31 January 2022

(C) The Japan Section of the Regional Science Association International 2022

\begin{abstract}
Articles included in the special feature section were explained in terms of Sustainable Development Goals (SDGs). It was shown that sustainability is conceptual and very flexible, while being too abstract for practice. This flexibility suggests a vast frontier for further research on Regional Sustainability in regional science fields. Various threats to humans were dealt with-global warming, natural disasters that could be intensified by global warming, the disappearance of marginal and minority communities, etc.
\end{abstract}

Keywords SDGs · Regional Sustainability · Global warming · Preparedness · Renewable energy $\cdot$ Disaster management $\cdot$ Energy intensity $\cdot$ Declining communities

\section{Sustainability}

This is an introductory article to the special feature section on Regional Sustainability. As expected, the included articles are diverse and it is hard to summarize them under one specific theme. We consider that this is due to the definition of sustainability. Given the huge volume of studies that deal with sustainability, obviously there exist different definitions - as many as the number of scholars and institutes.

Considering scientifically projected threats of climate change impacts on humans in the next 30 years, the highest priority is to verify whether sustainability holds whenever and wherever we carry out whatever action. However, sustainability is too general a concept, so that even conscious people become disoriented

Yoshiro Higano

higano@jsrsai.jp

1 University of Tsukuba, Tsukuba, Japan

2 Yokohama City University, Yokohama, Japan 
when trying to meet sustainability goals in practical settings. A well-known and primitive definition of sustainability may be given as follows: Current generations spend their life without causing any (additional) costs to future generations. If this holds for all the combinations of current and future generations over time, then we can say that sustainability holds (eternally) within the society of any generation in that time frame.

The definition of generation is flexible within the given time span within which the same generation exists. Precisely speaking, sustainability needs to hold with all the definitions of generation in the time frame. The definition of cost is also multifaceted. It is usually taken that costs mean environmental costs, because the global environmental issues in the coming three decades are so serious due to the imbalance between the enduring capacity of the Earth and outputs by society in any definition, especially in terms of greenhouse gas (GHG) emissions. However, generally speaking, this is not necessarily the case. It is natural to extend the definition of sustainability to incorporate passing costs, including those from "non-feasance" (omission) of different regions/countries/people with any definition even in the same generation, and so on. In a practical setting, sustainability is too abstract and comprehensive to use as a criterion or index to regulate, e.g., people's behavior. The definition of sustainability is a metaphor. Actually, considering the length of time allowable for humans, "without causing any (additional) costs" should now be replaced by "with compensating for the cost in the past (especially in terms of accumulated GHG emission)." However, we do not further discuss a possible alternative to "sustainability" because the point here is the last sentence in the first paragraph.

Changing the point of view, we can say that SDGs are specific and explicit goals that aim to induce codes for the behavior of the generation until 2030, in order to construct a sustainable society, focusing on current and near-future urgent global issues that should definitely be fixed by then. Implicit/explicit concepts and philosophy are fairness, equity, safety, peace (no conflict), cooperation, etc., which are necessary for the survival of humans. As mentioned with the (possible) variation in the definition of sustainability, nothing is said about costs that were caused and passed by the previous generations to the current generation. In analogy with the expansion of the definition above, cases in which the past generation passed costs to the current generation of different countries are serious. To construct a sustainable society, international collaboration is essential, but this would be difficult to establish without a clear solution for cost-bearing among different generations and/or different countries/regions. However, for the current generation, which is responsible to the future generation in terms of sustainability in any sense, collaboration is a "must" in a different sense than, e.g., compensation, subsidization, etc. The achievement of SDGs is a prerequisite for the construction of a sustainable or pseudosustainable society, and in this sense, SDGs are more practically oriented.

Accepting the concept and philosophy of sustainability and, therefore, varieties and ambiguity of the definition in the practical sense, we briefly explain articles in the special feature section, focusing on their positioning in achieving SDGs and their possible contribution to the expansion of frontiers of regional science. 


\section{Energy efficiency and substitution}

A substantial solution for the construction of a sustainable society, in terms of reducing GHG emissions and the threat due to climate change, is the conversion of the current energy system, which is highly dependent on fossil energy sources, to a renewable energy system. Effendi and Resosudarmo (2021) deals with the concern about continual increases in GHG emissions due to the utilization of fossil fuels to meet increasing demand for electricity in the Association of Southeast Asian Nations (ASEAN) countries. Considering the renewable energy sources of wind, solar, and hydro, they examined the impacts of diverting energy sources from fossil fuel-oriented into renewable energy-oriented on the ASEAN economy, based on the international/regional input-output analysis. Their research interests are whether the total GHG emission by the ASEAN countries can eventually be decreased by increasing the percentage of renewable energy sources; whether the growth of ASEAN economy is inevitably slower due to the utilization of renewable electricity, and whether the balanced growth in the ASEAN economy can be maintained through the spillover effects due to changes in the input-output structure of producing electricity, etc. The ASEAN countries are mostly developing countries, and it is a natural concern for them to maintain economic growth, while a top priority should be given to decreasing GHG emissions. ASEAN must be a cooperative body, and they are seriously concerned about disparities among constituent countries/regions. Using data that reflect different socioeconomic features of ASEAN countries/regions, interesting results are shown and suggested for scholars interested in the ASEAN economic community as a trade bloc, as well as the carbon emission trading among them.

The aim of the Paris Agreement is to make global GHG emissions peak by midcentury, and after a peak has been attained it must be offset by carbon sinks by drawing on forest absorption, carbon capture and storage (CCS) technology, etc. A major solution to address this goal is, of course, transformation of the energy system, which requires cutting-edge technology development. In this sense, it is effective to improve the energy efficiency of production as well as consumption, with fossil-oriented, hybrid, carbon neutral, or any energy system, to make the peak as early as possible before mid-century. Otsuka (2022) analyzed the impacts of improvements to the interregional transportation network of multimodes on the energy intensity in 47 prefectures in Japan. The construction of high-speed railways, such as Linear Chuo Shinkansen, is a national project that aims to enhance the modal shift from passenger vehicles and airplanes of high-energy intensity to public transport of low-energy intensity, and to provide efficient transportation infrastructures and modes to the local economy and people in marginal regions that are declining due to depopulation and aging. Research interests were whether improvements in the transportation network induced improvements in the energy intensity of passenger vehicles in 1990-2014; whether the high-speed railway improves the energy intensity of passenger vehicles indirectly through network improvement effects and directly through possible modal shifts; how the spatial energy intensities will be affected by network improvements in the future; 
whether GHG emissions are reduced by the high-speed railway construction, etc. Specifying the econometric model of novelty, the study firstly proved that a threshold exists for network improvement in terms of the time and distance of passenger travel in Japan, which leads to energy intensity improvement through modal shifts in passenger trips and changes in the spatial distribution of trip patterns among modes. Further research interests would be a comparison between the network improvement effects of Shinkansen (average speed $200 \mathrm{~km} / \mathrm{h}$ ) that was a cutting-edge transportation technology in the 1960s and Linear Chuo Shinkansen (more than $400 \mathrm{~km} / \mathrm{h}$ ) in 2020, on the regional development pattern.

\section{Exacerbated damage and preparedness}

The IPCC has reported that an increase in average temperatures by $1.5{ }^{\circ} \mathrm{C}$ in 2030-2050 is a critical threshold for the realization of threats to life on Earth, and the average temperature has already increased by $1.0^{\circ} \mathrm{C}$. While substantial solutions must be made through international agreement and collaboration, we must prepare for the threats of climate change, since extreme weather has recently affected lives and properties around the world, and we have possibly gone beyond a true critical threshold before the average temperature increase has reached the $1.5{ }^{\circ} \mathrm{C}$ IPCC projection. Threats or more specific damage by climate change can be divided into two categories: (1) direct threats due to heavy rain, storms, extreme heat or cold, typhoons, tornadoes, etc., and sea level rise, which could be taken as a specific phenomena caused by global warming; and (2) indirect threats due to the vulnerability of lives and properties from natural disaster, which could increase due to global warming. A typical example of the latter is damage caused by tidal waves. This increases when a typhoon occurs and is further exacerbated when the sea level has risen due to global warming. Other examples include damage caused by tsunamis when the sea level has risen, damage caused by avalanches triggered by earthquakes after heavy rain, etc. Most of these events, which are ascribable to climate change, are caused by unusual and unforeseeable events.

Aygün Oğur and Baycan (2022) highlighted that regional and urban planning, which is generally adopted by municipalities presuming ordinary and stochastically foreseeable events based on past experience, must be reconstructed by incorporating preparedness against the impacts of climate change on regional and urban systems. As the forecasted damages mostly occurred in coastal cities, a case study was developed with Istanbul in Turkey. The urban system is very complicated, and the current urban planning covers diverse sectors and services. The authors try to identify the threats from climate change against sectors and services, which are broken down into risk and vulnerability based on interview surveys conducted with experts. Prioritized sectors and services were identified with the Istanbul urban planning system. Climate change preparedness requires comprehensive strategic urban planning. However, in reality, the current urban planning itself is a tangled system related to the administrative system. In this sense, the identified priorities are useful and provide effective warnings for the administration units. 
Shibusawa and Matsushima (2022) provide useful projections and assessments for the preparedness of regional economies against natural disasters such as mega earthquakes and triggered tsunamis, which could be combined with possible nuclear accidents. The proposed model is based on a dynamic interregional input-output model generally applicable to any region, which was applied to 35 municipalities of Shizuoka Prefecture, Japan. Assuming noncompetitive import, their research interests are to identify vulnerable regions (municipalities) in terms of the rate of production recovery in the short term and speed of recovery in the long term, to the original production level; what conditions improve the identified vulnerability; how other factors, e.g., future aging and depopulation in Japan, affect the projected recovery scenario, etc. Generally speaking, this kind of assessment is dependent on the predictions of when the natural disaster occurs, and of course, such prediction is very difficult, and even causes other social issues. Owing to the linearity of the model and the incorporation of impacts into the specified dynamic interregional input-output model, the proposed assessment method is free from the prediction, as far as the scope of time horizon is concerned, for which the model specification is acceptable for utilization in this assessment.

Bangladesh is vulnerable to river and monsoon flooding, as two-thirds of the land is less than $5 \mathrm{~m}$ below sea level. This flooding seems to be worse recently due to global warming and/or extreme weather. The provision of a reliable transportation network is a prerequisite for the sustainable development of the regional economy. However, annual floods interrupt the stable flow of traffic on the national highway network, and the estimated loss due to floods is around $0.7 \%$ of the gross domestic product (GDP). Choudhury et al. (2022) have tried to provide a reliable index with which a limited budget for maintenance of the national highway can be efficiently allocated to the sections of the network that have been most affected by floods and have a high risk of disconnection. The study proposed an index of "flood-affected road length," which can be utilized for the efficient allocation of a limited budget. Seven critical floods were selected from flood events that occurred from 2003 to 2017 and, using geographic information system/remote sensing (GIS/RS) data, the national highway sections that were attacked by the seven floods are accumulated into the flood-affected road length - an index of the risk of disconnection due to flooding, because manually collected data of previous flood events from several sources are sometimes not reliable due to reasons pertinent to developing countries.

\section{Regional economic growth and happiness}

There was a shocking article in a major newspaper that predicts 896 among 1800 municipalities will possibly cease to exist by 2040 due to the declining birth rate in Japan. If this is the case, from the financial view of municipalities, the situation becomes worse due to the aging of population. Of course, the prediction is subject to "if no countermeasures are taken," which is not acceptable in any manner from the sustainability requirement of SDGs: economic growth, employment opportunities, and a happy life for everybody. Having observed a decline in the domestic supply of primary and food/beverage industries under a mild depopulation period from 2000 
to 2015, Tokunaga and Okiyama (2022) proposed countermeasures of a demandside consumption tax reduction policy and a supply-side innovative agrobased food industry cluster policy to sustain primary industries in the upcoming rapid depopulation period from 2015 to 2030. Using a regional computable general equilibrium model, it was shown that a consumption tax reduction policy and a supply-side innovative agrobased food industry cluster policy are effective in sustaining the national economy in the short and long term, respectively. However, depending on concentration of medium-sized factories and the population, especially in the Kanto District where Tokyo is located, the policy impacts vary by region. This means further countermeasures must be prescribed for marginal regions since depopulation combined with aging is a critical issue in Japan.

Ethnic tourism has been strategically developed for regional economic development in many countries. It is generally believed, and taken for granted, that it sustains the preservation of unique and invaluable cultures and traditions, especially for the heritage of minority groups of local inhabitants. The United Nations' World Tourism Organization states that sustainable tourism should enhance sustainability in economic, social, environmental, and cultural fields. Mayuzumi (2022) focuses on tourism with the traditional culture of Balinese sculptures and paintings in Bali, and examines if ethnic tourism would eventually bring happiness to related people in terms of livelihood status, as well as preserving their proud traditional cultures. This work suggests preservation of cultural heritage should not be directly combined with tourism. Therefore, we need further research on social and cultural policy, which should be properly prescribed considering the features of the region and the consciousness of the people involved.

\section{Conclusions}

In this introductory article to the special feature section on Regional Sustainability, the focus and interest of each article is explained in light of the SDGs, as sustainability itself is a general and comprehensive concept and is too abstract to be adopted into practices such as sustainable regional and urban planning. A possible vast frontier of regional science is suggested for the comprehensive study of threat: not being sustainable (being against sustainability) should now be taken as a threat against humans.

\section{References}

Aygün Oğur A, Baycan T (2022) Identifying priority planning areas of Istanbul for climate change preparedness. Asia-Pac J Reg Sci. https://doi.org/10.1007/s41685-021-00225-4

Choudhury KN, Yabar H, Mizunoya T (2022) GIS and remote sensing-based spatiotemporal analysis of cumulative flood risk over Bangladesh's national highways. Asia-Pac J Reg Sci. https://doi.org/10. 1007/s41685-021-00216-5

Effendi Y, Resosudarmo BP (2021) Development of renewable electricity in ASEAN countries: socio-economic and environmental impacts. Asia Pac J Reg Sci. https://doi.org/10.1007/ s41685-021-00206-7 
Mayuzumi Y (2022) Is meeting the needs of tourists through ethnic tourism sustainable? Focus on Bali, Indonesia. Asia-Pac J Reg Sci. https://doi.org/10.1007/s41685-021-00198-4

Otsuka A (2022) Assessment of the improvement in energy intensity by the new high-speed railway in Japan. Asia-Pac J Reg Sci. https://doi.org/10.1007/s41685-020-00165-5

Shibusawa H, Matsushima D (2022) Assessing the economic impact of tsunami and nuclear power plant disasters in Shizuoka, Japan: a dynamic inter-regional input-output (IRIO) approach. Asia-Pac J Reg Sci. https://doi.org/10.1007/s41685-021-00196-6

Tokunaga S, Okiyama M (2022) Impact of changes in the labor force and innovative agro-based food industry clusters on primary and food-beverage industries, and regional economies in Japan's depopulating society. Asia-Pac J Reg Sci. https://doi.org/10.1007/s41685-021-00210-x

Publisher's Note Springer Nature remains neutral with regard to jurisdictional claims in published maps and institutional affiliations. 